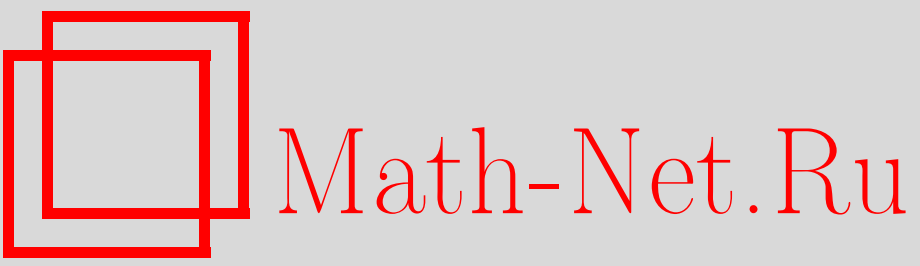

Ф. Розенхаус, О существенных законах сохранения для уравнений с бесконечными симметриями, ТМФ, 2005, том 144, номер 1, 190-198

DOI: https://doi.org/10.4213/tmf1845

Использование Общероссийского математического портала Math-Net.Ru подразумевает, что вы прочитали и согласны с пользовательским соглашением

http://www.mathnet.ru/rus/agreement

Параметры загрузки:

IP: 54.172 .240 .79

26 апреля 2023 г., $12: 16: 29$ 
ТЕОРЕТИЧЕСКАЯ

И МАТЕМАТИЧЕСКАЯ

ФИЗИКА

Том 144, № 1

июль, 2005

(C) 2005 r.

Ф. Розенхаус*

\section{О СУЩЕСТВЕННЫХ ЗАКОНАХ СОХРАНЕНИЯ ДЛЯ УРАВНЕНИЙ С БЕСКОНЕЧНЫМИ СИММЕТРИЯМИ}

Рассматриваются дифференциальные уравнения в частных производных для вариационной задачи с бесконечной группой симметрии. Исследуются локальные законы сохранения, связанные с произвольными функциями одной переменной от генераторов группы. Показано, что только симметрии с произвольными функциями зависимых переменных приводят к бесконечному числу законов сохранения. Вычислены локальные законы сохранения для потенциального уравнения Заболоцкой-Хохлова для одной из его бесконечных подгрупп.

Ключевые слова: бесконечные симметрии, законы сохранения.

\section{1. ВВЕДЕНИЕ}

Вопрос о взаимоотношении симметрий и законов сохранения имеет давнюю историю, восходящую к знаменитой работе Нётер [1]. Первая теорема Нётер устанавливает соответствие между $r$-параметрической группой Ли симметрий функционала действия и $r$ локальными законами сохранения. Согласно второй теореме Нётер бесконечные вариационные симметрии (с произвольными функциями всех независимых переменных, см., например, [2]) приводят к соотношениям между уравнениями исходной дифференциальной системы, а не к законам сохранения. Обший случай бесконечных симметрий с произвольными функциями от $k<n$ независимых переменных в $\mathbb{R}^{n}$ был исследован в [2]. В настоящей работе мы рассмотрим лагранжевы дифференциальные уравнения, допускающие бесконечную алгебру симметрий с операторами, зависящими от произвольной функции независимых и зависимых переменных и их производных. Используя развитый в [2] подход, мы вычисляем некоторые локальные сохраняющиеся величины для трехмерного потенциального уравнения Заболоцкой-Хохлова и обсуждаем роль граничных условий. Будет показано, что только произвольные функции зависимых переменных от генераторов группы симметрии могут приводить к бесконечному числу законов сохранения.

*Department of Mathematics and Statistics, California State University, Chico, CA 95929, USA. E-mail: vrosenhaus@csuchico.edu 


\section{2. ЗАКОНЫ СОХРАНЕНИЯ И ТЕОРЕМЫ НЁТЕР}

Под законом сохранения для заданного дифференциального уравнения

$$
\omega\left(x, u, u_{i}, \ldots\right)=0
$$

понимается уравнение непрерывности

$$
D_{\mu} K_{\mu} \doteq 0, \quad \mu=1, \ldots, n,
$$

выполненное для любых решений уравнения (1). Каждый закон сохранения определен с точностью до преобразования эквивалентности $K_{\mu} \rightarrow K_{\mu}+P_{\mu}, D_{\mu} P_{\mu}=0$. Следующие случаи приводят к тривиальным законам сохранения [3]:

1) $D_{\mu} K_{\mu}=0$ : уравнение непрерывности выполнено во всем пространстве;

2) $K_{\mu} \doteq 0(\mu=1, \ldots, n):$ на решениях уравнения (1) компоненты вектора $K_{\mu}$ обрашаются в нуль.

Под существенным законом сохранения мы будем понимать такой нетривиальный закон сохранения $D_{\mu} K_{\mu} \doteq 0$, который приводит к ненулевой сохраняющейся величине

$$
D_{t} \iiint K_{t} d x d y d z \doteq 0, \quad K_{t} \neq 0
$$

Пусть

$$
S=\int L\left(x^{i}, u, u_{i}, \ldots\right) d^{n} x
$$

- функционал действия, $x^{i}=(x, y, \ldots, t)$ - независимые переменные и $u_{i} \equiv \partial u(x) / \partial x^{i}$, $i=1, \ldots, n$. Уравнения движения имеют вид

$$
E(L) \equiv \omega\left(x, u, u_{i}, u_{i j}, \ldots\right)=0
$$

где $E$ - оператор Эйлера-Лагранжа,

$$
E=\frac{\partial}{\partial u}-D_{i} \frac{\partial}{\partial u_{i}}+\sum_{i \leqslant j} D_{i} D_{j} \frac{\partial}{\partial u_{i j}}+\cdots
$$

Рассмотрим инфинитезимальное преобразование [4], связанное с оператором

$$
X=\xi^{i} \frac{\partial}{\partial x^{i}}+\eta \frac{\partial}{\partial u}+\zeta^{i} \frac{\partial}{\partial u_{i}}+\cdots
$$

и с соответствующим каноническим оператором

$$
X_{\alpha}=\alpha \frac{\partial}{\partial u}+\sum_{i}\left(D_{i} \alpha\right) \frac{\partial}{\partial u_{i}}+\sum_{i \leqslant j}\left(D_{i} D_{j} \alpha\right) \frac{\partial}{\partial u_{i j}}+\cdots, \quad i, j=1, \ldots, n,
$$

где $\alpha=\eta-\xi^{i} u_{i}$. 
Используем тождество Нётер [2]

$$
X_{\alpha}=\alpha E+\sum_{i=1} D_{i} R_{\alpha i},
$$

где

$$
R_{\alpha i}=\alpha \frac{\partial}{\partial u_{i}}+\left\{\sum_{k \geqslant i}\left(D_{k} \alpha\right)-\alpha \sum_{k \leqslant i} D_{k}\right\} \frac{\partial}{\partial u_{i k}}+\cdots
$$

Вариация функционала $S$ задается формулой

$$
\delta S=\int\left[X_{\alpha} L+D_{i}\left(\xi^{i} L\right)\right] d^{n} x .
$$

Пусть преобразование $X_{\alpha}$ есть вариационная (нётеровская) симметрия,

$$
X_{\alpha} L=D_{i} M_{i} .
$$

Применение тождества Нётер (8) к $L$ дает

$$
X_{\alpha} L=\alpha \omega+D_{i}\left(R_{\alpha i} L\right) .
$$

Сравнивая два последних равенства, приходим к соотношению

$$
D_{i}\left(M_{i}-R_{\alpha i} L\right)=\alpha \omega,
$$

т.е. к уравнению непрерывности на многообразии решений $\left(\omega=0, D_{i} \omega=0, \ldots\right)$

$$
D_{i}\left(M_{i}-R_{\alpha i} L\right) \doteq 0 .
$$

В соответствии с этим уравнением любая вариационная симметрия $\alpha$ в случае конечной группы Ли приводит к соответствуюшему закону сохранения (первая теорема Нётер [1]). Вторая теорема Нётер [1] имеет дело со случаем бесконечной группы вариационных симметрий, где вектор симметрии $\alpha$ имеет вид

$$
\alpha=a p(x)+b_{i} D_{i} p(x)+c_{i j} D_{i} D_{j} p(x)+\cdots,
$$

и $p(x)$ является произвольной функцией всех базисных пространственных переменных. В отличие от первой теоремы Нётер, следствием бесконечной симметрии функционала $\omega$ в уравнении (1) является не закон сохранения, а некоторое соотношение между исходными дифференциальными уравнениями [1]:

$$
a^{b} \omega^{b}-D_{i}\left(b_{i}^{b} \omega^{b}\right)+D_{i} D_{j}\left(c_{i j}^{b} \omega^{b}\right)+\cdots=0,
$$

где $a^{b}, b_{i}^{b}, c_{i j}^{b}, \ldots$ - компоненты вектора симметрии $\alpha(13)$. Общий случай, когда $p(x)$ есть произвольная функция не всех базисных переменных, был исследован в работе [2]. В настоящей работе нас интересует случай произвольных функций одной переменной. 


\section{3. НЁТЕРОВСКИЕ И СТРОГИЕ ГРАНИЧНЫЕ УСЛОВИЯ}

Рассмотрим функции $u=u(x)$, определенные на области $D \subset \mathbb{R}^{m+1}$. Пусть симметрия $\alpha$ дифференциального уравнения с функцией Лагранжа $L=L\left(x^{i}, u, u_{i}\right)$ имеет вид

$$
\alpha=a \gamma(\theta)+b \gamma^{\prime}(\theta)+c \gamma^{\prime \prime}(\theta)+\cdots+h \gamma^{(l)}(\theta), \quad \theta=\theta\left(x, u, u_{i}\right) .
$$

Для нётеровского преобразования симметрии $X_{\alpha}$ имеем

$$
\delta S=\int \cdots \int \delta L d^{m+1} x=\int \cdots \int X_{\alpha} L d^{m+1} x=\int \cdots \int D_{i} M_{i} d^{m+1} x=0 .
$$

Поэтому должны быть выполнены следуюшие условия на $M_{i}$ (нётеровские граничные условия) [2], [5]:

$$
\left.M_{i}(x, u, \ldots)\right|_{x^{i} \rightarrow \partial D}=0 \quad \forall i=1, \ldots, m+1 .
$$

Эти условия обычно выполнены в случае "регулярного" асимптотического поведения, $u, u_{i} \rightarrow 0$ при $x \rightarrow \pm \infty$, или для периодических решений. Мы рассмотрим граничные условия другого типа, связанные с сушествованием локально сохраняющихся величин. Интегрируя уравнение (12) по пространству переменных $x^{1}, x^{2}, \ldots, x^{m}$ и ограничиваясь многообразием решений, получаем

$$
\int \ldots \int d x^{1} \ldots d x^{m} D_{t}\left(M_{t}-R_{\alpha t} L\right) \doteq \int \ldots \int d x^{1} \ldots d x^{m} \sum_{i=1}^{m} D_{i}\left(R_{\alpha i} L-M_{i}\right) .
$$

Применяя нётеровское граничное условие (16) и требуя, чтобы левая часть последнего уравнения обрашалась в нуль на многообразии решений, получаем "строгие" граничные условия [2], [5]

$$
\left.R_{\alpha 1} L\right|_{x^{1} \rightarrow \partial D}=\left.R_{\alpha 2} L\right|_{x^{2} \rightarrow \partial D}=\cdots=\left.R_{\alpha m} L\right|_{x^{m} \rightarrow \partial D}=0 .
$$

В случае $L=L\left(x, u, u_{j}\right)$ строгие граничные условия (18) принимают простой вид

$$
\left.\alpha \frac{\partial L}{\partial u_{i}}\right|_{x^{i} \rightarrow \partial D}=0 \quad \forall i=1, \ldots, m .
$$

Для того чтобы система обладала локальными сохраняюшимися величинами, должны быть выполнены и нётеровские (16), и строгие (18) граничные условия. В этом случае можно найти соответствующий нётеровский закон сохранения

$$
\int \cdots \int d x^{1} \ldots d x^{m} D_{t}\left(M_{t}-R_{\alpha t} L\right) \doteq 0 .
$$

Записывая $M_{t}$ в виде

$$
M_{t}=A \gamma(\theta)+B \gamma^{\prime}(\theta)+C \gamma^{\prime \prime}(\theta)+\cdots+H \gamma^{(l)}(\theta), \quad \theta=\theta(x),
$$

и используя уравнения (21) и (15), получаем

где

$$
D_{t} \int \cdots \int d x^{1} \ldots d x^{m}\left[\gamma(\theta) a_{0}+\gamma^{\prime}(\theta) a_{1}+\cdots+\gamma^{(l)}(\theta) a_{l}\right] \doteq 0,
$$

$$
a_{0}=A-a \frac{\partial L}{\partial u_{t}}, \quad a_{1}=B-b \frac{\partial L}{\partial u_{t}}, \quad \ldots, \quad a_{l}=H-h \frac{\partial L}{\partial u_{t}}, \quad i=0, \ldots, l .
$$




\section{4. НЕСИНГУЛЯРНОЕ ПРОСТРАНСТВЕННОЕ \\ ПРЕОБРАЗОВАНИЕ $\theta=\theta(x)$}

Введем новые переменные интегрирования, совершив замену $\left(x^{1}, x^{2}, \ldots, x^{m}\right) \stackrel{\psi}{\longrightarrow}$ $\left(x^{1}, \ldots, x^{p-1}, \theta, x^{p+1}, \ldots, x^{m}\right)$. В случае несингулярного преобразования $\psi$ можно обратить соотношение $\theta=\theta\left(x^{1}, x^{2}, \ldots, x^{m}, t\right)$ как

$$
x^{p}=\varphi^{p}\left(x^{1}, \ldots, x^{p-1}, \theta, x^{p+1}, \ldots, x^{m}, t\right)
$$

и на основании равенства (22) получить, что

$$
D_{t} \int \ldots \int d \theta d x^{1} \ldots d x^{p-1} d x^{p+1} \ldots d x^{m} \sum_{i=0}^{l} \bar{a}_{i} \gamma^{(i)}(\theta) \doteq 0
$$

где

$$
\gamma^{(i)}(\theta)=\frac{\partial^{i} \gamma(\theta)}{\partial \theta^{i}}, \quad \bar{a}_{i}=a_{i} \frac{\partial \varphi^{p}}{\partial \theta}, \quad i=0, \ldots, l, \quad p=1, \ldots, m,
$$

и $J=\partial \varphi^{p} / \partial \theta$ - якобиан преобразования $\psi$. Возьмем интеграл (23) по частям и выберем произвольно функцию $\gamma(\theta)$, обрашаюшуюся в нуль на границе вместе со своими производными всех порядков до $l-1$ :

$$
\left.\gamma(\theta)\right|_{\theta \rightarrow \partial D}=\left.\gamma^{\prime}(\theta)\right|_{\theta \rightarrow \partial D}=\left.\gamma^{\prime \prime}(\theta)\right|_{\theta \rightarrow \partial D}=\cdots=\left.\gamma^{(l-1)}\right|_{\theta \rightarrow \partial D}=0
$$

Тогда получим

$$
D_{t} \int \ldots \int d \theta d x^{1} \ldots d x^{p-1} d x^{p+1} \ldots d x^{m} \gamma(\theta) \sum_{i=0}^{l}(-1)^{i} \partial_{\theta}^{i} \bar{a}_{i} \doteq 0
$$

Поскольку функция $\gamma(\theta)$ произвольна, можно на основе известной теоремы анализа [6] прийти к выводу, что

$$
D_{t} \int \ldots \int d x^{1} \ldots d x^{p-1} d x^{p+1} \ldots d x^{m}\left[\bar{a}_{0}-\partial_{\theta} \bar{a}_{1}+\partial_{\theta}^{2} \bar{a}_{2}+\cdots+(-1)^{l} \partial_{\theta}^{l} \bar{a}_{l}\right] \doteq 0
$$

Это уравнение дает выражение для сохраняющейся величины, соответствуюшей бесконечной симметрии $\alpha$ вида (15) при условии, что вьполнены граничные условия (16), (19) и (25). Таким образом, бесконечная алгебра симметрий (15) с произвольной функцией независимых переменных в случае несингулярного преобразования приводит в точности к одному закону сохранения (27). 


\section{5. ПРОИЗВОЛЬНЫЕ ФУНКЦИИ ВРЕМЕНИ}

Если $\partial \theta / \partial x^{p}=0$ для любого $p \in(1, \ldots, m)$, то $\theta=t$. Аналогично уравнению $(22)$ получаем

$$
D_{t} \int \cdots \int d x^{1} \ldots d x^{m}\left[\gamma(t) a_{0}(t)+\gamma^{\prime}(t) a_{1}(t)+\cdots+\gamma^{(l)}(t) a_{l}(t)\right] \doteq 0 .
$$

Поскольку $\gamma(t)$ - произвольная функция, имеем

$$
\begin{aligned}
\int \ldots \int d x^{1} \ldots d x^{m} a_{0}(t) & \doteq \int \cdots \int d x^{1} \ldots d x^{m} a_{1}(t) \doteq \cdots \\
\ldots & \doteq \int \cdots \int d x^{1} \ldots d x^{m} a_{l}(t) \doteq 0 .
\end{aligned}
$$

Уравнения (29), вообше говоря, определяют систему не законов сохранения, а дополнительных связей. Существуют три возможности.

1. Строгие и нётеровские граничные условия (18), (16) могут быть выполнены для произвольной функции $\gamma(t)$. Никакие законы сохранения не связанны с такой симметрией.

2. Строгие и нётеровские граничные условия могут быть выполнены для некоторых конкретных функций $\gamma(t)$. Каждый выбор функции $\gamma(t)$ приводит к соответствуюшей сохраняюшейся величине (20).

3. Строгие граничные условия не могут быть выполнены ни для каких функций $\gamma(t)$. В таком случае следствием бесконечной симметрии является тот факт, что решений исходного дифференциального уравнения с граничными условиями (18), (16) не существует.

Применим изложенный выше подход к поиску существенных законов сохранения для потенциального уравнения Заболоцкой-Хохлова [7]

$$
u_{x t}-u_{x} u_{x x}-u_{y y}-u_{z z}=0 .
$$

Функция Лагранжа этого уравнения имеет вид

$$
L=-\frac{u_{x} u_{t}}{2}+\frac{u_{x}^{3}}{6}+\frac{u_{y}^{2}}{2}+\frac{u_{z}^{2}}{2} .
$$

Рассмотрим бесконечную подгруппу точечной группы Ли симметрий уравнения (30), связанную с оператором

$$
X_{\gamma}=-\gamma(t) \frac{\partial}{\partial x}+\left[\gamma^{\prime}(t) x+\gamma^{\prime \prime}(t) \frac{y^{2}+z^{2}}{4}\right] \frac{\partial}{\partial u},
$$

где $\gamma(t)$ - произвольная функция (свойства симметрии уравнения Заболоцкой-Хохлова изучались в работах $[8])$. Имеем

$$
\begin{array}{ccc}
\alpha=\gamma^{\prime} x+\gamma^{\prime \prime} \frac{y^{2}+z^{2}}{4}+\gamma u_{x}, \quad X_{\alpha} L=D_{i} M_{i}, & M_{t}=-\frac{u \gamma^{\prime}}{2}, \\
M_{x}=\gamma L-\frac{x u \gamma^{\prime \prime}}{2}-u \gamma^{\prime \prime \prime} \frac{y^{2}+z^{2}}{8}, \quad M_{y}=\frac{y u \gamma^{\prime \prime}}{2}, & M_{z}=\frac{z u \gamma^{\prime \prime}}{2}, \\
a=u_{x}, \quad b=x, \quad c=\frac{y^{2}+z^{2}}{4}, & A=C=0, & B=-\frac{u}{2} .
\end{array}
$$


Нётеровские и строгие граничные условия зависят от функции $\gamma(t)$.

Случай $А$. Пусть функция $\gamma(t)$ произвольна. Нётеровские условия (16) для $X_{\alpha}$ имеют вид

$$
u_{i} \underset{x \rightarrow \pm \infty}{\longrightarrow} 0, \quad x u \underset{x \rightarrow \pm \infty}{\longrightarrow} 0, \quad y u \underset{y \rightarrow \pm \infty}{\longrightarrow} 0, \quad z u \underset{z \rightarrow \pm \infty}{\longrightarrow} 0, \quad u \underset{t \rightarrow \pm \infty}{\longrightarrow} 0 .
$$

Строгие условия (19) принимают вид

$$
x u_{t}, x u_{x}^{2} \underset{x \rightarrow \pm \infty}{\longrightarrow} 0, \quad y^{2} u_{y} \underset{y \rightarrow \pm \infty}{\longrightarrow} 0, \quad z^{2} u_{z} \underset{y \rightarrow \pm \infty}{\longrightarrow} 0 .
$$

Никакие локальные законы сохранения не связаны с нётеровским преобразованием $X_{\alpha}$ $(32)$ для произвольной $\gamma(t)$. Дополнительные связи (29) приводят только к тривиальному решению. Сушествование бесконечной симметрии (32) приводит к отсутствию нетривиальных решений, удовлетворяющих граничным условиям (34), (35).

Рассмотрим теперь некоторые конкретные функции $\gamma(t)$, для которых можно ослабить наши граничные условия (34), (35), чтобы избежать ограничений (29).

Случай Б. Пусть $\gamma^{\prime}(t)=0, \gamma(t)=c=$ const. Нётеровские условия имеют вид

$$
u_{i} \underset{x \rightarrow \infty}{\longrightarrow} 0
$$

Для “строгих” граничных условий в дополнение нётеревским условиям имеем

$$
u_{x} u_{y} \underset{y \rightarrow \pm}{\longrightarrow} 0, \quad u_{x} u_{z} \underset{z \rightarrow \pm \infty}{\longrightarrow} 0 .
$$

Согласно (20) получаем закон сохранения

$$
D_{t} \iiint u_{x}^{2} d x d y d z \doteq 0
$$

Соответствующее условию (35) уравнение непрерывности записывается как

$$
D_{x}\left(-\frac{u_{x}^{3}}{3}+\frac{u_{y}^{2}}{2}+\frac{u_{z}^{2}}{2}\right)+D_{y}\left(-u_{x} u_{y}\right)+D_{z}\left(-u_{x} u_{y}\right)+D_{t}\left(\frac{u_{x}^{2}}{2}\right) \doteq 0 .
$$

Случай $B$. Пусть $\gamma^{\prime \prime}(t)=0, \gamma(t)=a t, a=\mathrm{const} \neq 0$. Нётеровские условия имеют вид

$$
u_{i} \underset{x \rightarrow \pm \infty}{\longrightarrow} 0, \quad u \underset{t \rightarrow \pm \infty}{\longrightarrow} 0 .
$$

В дополнение к ним для строгих граничных условий имеем

$$
x u_{t}, x u_{x}^{2} \underset{x \rightarrow \pm \infty}{\longrightarrow} 0, \quad u_{y}, u_{x} u_{y} \underset{y \rightarrow \pm \infty}{\longrightarrow} 0, \quad u_{z}, u_{x} u_{z} \underset{z \rightarrow \pm \infty}{\longrightarrow} 0 .
$$

Сушественный закон сохранения (20), связанный с граничными условиями (39), (40), принимает вид

$$
D_{t} \iiint\left[x u_{x}-u+t u_{x}^{2}\right] d x d y d z \doteq 0 .
$$

Случай $\Gamma$. Пусть $\gamma^{\prime \prime}(t) \neq 0$. В этом случае получаем те же граничные условия (34), (35), что и в случае А, и те же дополнительные связи, не оставляющие никаких нетривиальных решений. Анализ других бесконечных подалгебр уравнения (30) будет приведен в отдельной публикации. 


\section{6. ПРОИЗВОЛЬНЫЕ ФУНКЦИИ ОТ $u, u_{i}$}

Рассмотрим симметрию

$$
\alpha=a f(\theta)+b f^{\prime}(\theta)+c f^{\prime \prime}(\theta)+\cdots+h f^{(l)}(\theta), \quad \theta=\theta\left(u, u_{i}\right),
$$

где $f=f(\theta)$ - произвольная функция. Мы предполагаем, что имеют место регулярное асимптотическое поведение $u, u_{i} \underset{x^{i} \rightarrow \pm \infty}{\longrightarrow} 0$ и условие $\theta(0, \ldots, 0)=a$. Тогда как нетеровские (16), так и строгие (19) граничные условия выполнены, если

$$
f(a)=f^{\prime}(a)=f^{\prime \prime}(a)=\cdots=f^{(l)}(a)=0 .
$$

Согласно формуле (20) получаем набор сохраняющихся величин с произвольной функцией $f(\theta)$, удовлетворяющей равенствам (43) и нётеровскому граничному условию (16). Таким образом, случай нётеровской симметрии с произвольной функцией от $u, u_{i}$ может приводить к бесконечному набору законов сохранения. В качестве примера такой ситуации рассмотрим следующее уравнение:

$$
v_{x t}-v_{x} v_{x x}=0
$$

Функция Лагранжа этого уравнения есть

$$
L=\frac{v_{x} v_{t}}{2}+\frac{v_{x}^{3}}{6}
$$

Уравнение (44) инвариантно относительно бесконечной подгруппы с оператором

$$
X_{\varphi}=\varphi\left(v_{x}\right) \frac{\partial}{\partial v}
$$

где $\varphi=\varphi\left(v_{x}\right)-$ произвольная функция. Вычисляя $X_{\varphi} L$, получаем

$$
X_{\varphi} L=D_{x} M_{x}+D_{t} M_{t}, \quad M_{x}=\frac{\varphi\left(v_{x}\right) v_{t}-R\left(v_{x}\right)}{2}, \quad M_{t}=\frac{P\left(v_{x}\right)}{2}
$$

где

$$
P^{\prime}(z)=z \varphi^{\prime}(z)-\varphi(z), \quad R^{\prime}(z)=z^{2} \varphi^{\prime}(z) .
$$

Нётеровские граничные условия (16) для $X_{\varphi}$ можно записать как

$$
\left.M_{x}\left(x, t, u, u_{i}\right)\right|_{x \rightarrow \pm \infty}=\left.M_{t}\left(x, t, u, u_{i}\right)\right|_{t \rightarrow \pm \infty}=0 .
$$

Предположим, что имеет место регулярное асимптотическое поведение функции $v$ и ее производных

$$
v_{x}, v_{t} \underset{x \rightarrow \pm \infty}{\longrightarrow} 0, \quad v_{x} \underset{x \rightarrow \pm \infty}{\longrightarrow} 0 .
$$


Тогда нётеровские граничные условия выполнены, если

$$
\varphi(0)=P(0)=R(0)
$$

Строгие граничные условия (19) принимают вид

$$
\varphi\left(v_{x}\right)\left(v_{t}-v_{x}^{2}\right) \underset{x \rightarrow \pm \infty}{\longrightarrow} 0
$$

и это условие с очевидностью выполнено вследствие (49) и (50). Бесконечный набор локальных законов сохранения (20), связанных с симметрией (46), описывается следующей формулой:

$$
D_{t} \int\left[P\left(v_{x}\right)-v_{x} \varphi\left(v_{x}\right)\right] d x \doteq 0
$$

где $\varphi=\varphi\left(v_{x}\right)$ - произвольная функция, $P=P\left(v_{x}\right)$ удовлетворяет первому из уравнений (48).

\section{7. ЗАКЛЮЧЕНИЕ}

Бесконечные симметрии с произвольными функциями не всех независимых переменных приводят к конечному числу законов сохранения. Каждый сушественный закон сохранения связан с конкретной формой граничного условия. Бесконечные симметрии с произвольными функциями зависимых переменных и их производных могут приводить к бесконечному числу законов сохранения.

Благодарности. Я благодарен М. Павлову за полезные обсуждения.

\section{Список литературы}

[1] E. Noether. Invariantevariationsprobleme, Nachr. König. Gessell. Wissen. Göttingen, Math.Phys. Kl. 1918. P. 235.

[2] V. Rosenhaus. J. Math. Phys. 2002. V. 43. P. 6129.

[3] П. Олвер. Приложения групп Ли к дифференциальным уравнениям. М.: Мир, 1989.

[4] H. Х. Ибрагимов. Группы преобразований в математической физике. М.: Наука, 1983.

[5] V. Rosenhaus. One-dimensional infinite symmetries, boundary conditions and local conservation laws. In: Symmetry and Perturbation Theory - SPT2001. Proc. of the Int. Conf. SPT2001 (Gala Gonone, Sardinia, Italy, 6-13 May 2001). Eds. D. Bambusi, G. Gaeta, M. Cadoni. Singapore: World Scientific, 2001. P. 183.

[6] И. М. Гельфанд, С. В. Фомин. Вариационное исчисление. М.: Физматгиз, 1961.

[7] Е. А. Заболотская, Р. В. Хохлов. Акуст. журн. 1969. Т. 15. № 1. С. 40.

[8] А. М. Виноградов, И. С. Красильщик, В. В. Лычагин. Введение в геометрию нелинейных дифференциальных уравнений. М.: Наука, 1986; F. Schwarz. J. Phys. А. 1987. V. 20. Р. 1613. 